TECHNOLOGY FEATURE

\title{
Getting inside their minds
}

\begin{tabular}{ll} 
Built for speed & 774 \\
\hline Penetrating insights & 776 \\
\hline Seeing where the action is & 779 \\
\hline All together now & 780 \\
\hline Box 1: Form and function & 774 \\
\hline Box 2: On their best behavior & 778 \\
\hline
\end{tabular}

\author{
Michael Eisenstein
}

\section{Neuroscientists are taking advantage of powerful new tools for fluorescence imaging that enable detailed visualization of the structure and activity of neuronal circuits within the living brain.}

Although Bell Labs is probably best known for game-changing physical sciences breakthroughs such as the laser and the transistor, it has also given neuroscientists considerable cause for gratitude. It was there, in the early 1990s, that physicist Winfried Denk found the opportunity to collaborate with brain researchers on refining a powerful new technique for imaging based on excitation of fluorophores with focused, high-intensity pulses of long-wavelength laser light, which he had initially developed as a student with Watt Webb at Cornell University.

This approach, which Denk and Webb termed multiphoton or, more specifically, two-photon microscopy, offers a number of important advantages over confocal imaging for live-cell studies. The use of near-infrared beams rather than ultraviolet lasers allows deep sample penetration with reduced scattering and minimal cell damage. Furthermore, because imaging relies on the excitation of fluorophores via nearsimultaneous stimulation with multiple low-energy photons, fluorescence emission is localized to a highly restricted focal area, making every detectable photon useful and eliminating the need to block out-of-focus fluorescence.

These features proved a tremendous boon for observing activity inside the highly scattering tissues of the brain, and Columbia University neuroscientist Rafael Yuste was an early convert. "Denk and I worked together for the summer in 1993, and we got the first data from imaging living neurons with two-photon [excitation] deep within brain slices," he says. "Essentially, there was no going back after that."

Today, a growing number of essentially 'turnkey' commercial options for two-photon microscopy are helping to popularize a technology that was once the domain of do-it-yourself types, and Carl Zeiss, Olympus and Leica Microsystems are among the companies offering such platforms. "For neuroscience, I think that ultimately you'll see less and less confocal if you need depth of penetration," says Dennis Donley, group manager for laser scanning confocal microscopes at Olympus America. According to Donley, this was a primary consideration in developing the FluoView FV1000-MPE multiphoton system. "Our philosophy from the beginning was to get the deepest image," he says. "We wanted 700-800 microns down, and we've had customers go down to 1.1 millimeters." Other key features include the capacities to detect signals on up to four channels and to incorporate multiple lasers for simultaneous excitation at different wavelengths or for combined stimulationdetection experiments.

This dual-scanning capacity is also a key feature of the LSM 7 MP, launched last fall by Carl Zeiss MicroImaging. Unlike the older LSM 710 NLO system, which combines both confocal and multiphoton imaging capabilities, the LSM 7 MP was purpose-built for two-photon microscopy and, more specifically, for in vivo imaging (see "Penetrating insights" below). "You can have up to five different channels for imaging," says product manager Eva Simbürger. "And the scanner is designed for high transmission in that you get the laser to the sample and don't lose power. You don't want to lose energy you might need for the imaging process."

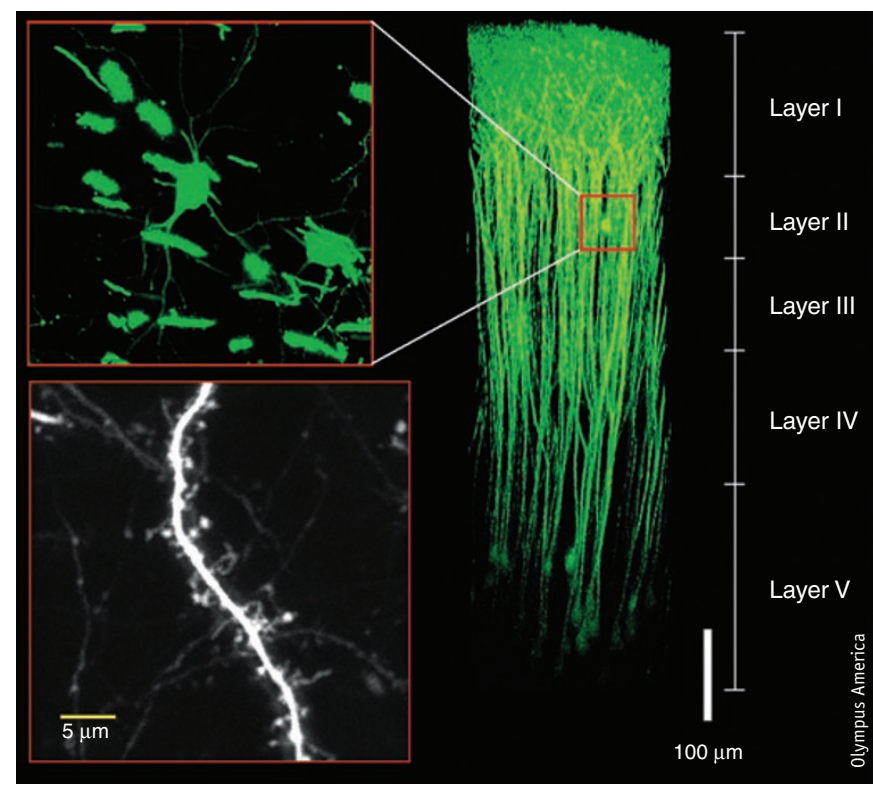

Hiroaki Waki, Tomomi Nemoto and Junichi Nabekura of Japan's National Institutes of Natural Sciences have used their Olympus FluoView FV1000-MPE to image neurons as deep as 700 micrometers within the cerebral neocortex of mice. 


\section{BOX 1 FORM AND FUNCTION}

It didn't take long for the neuroscientists at Petilla de Aragón to recognize their problem; they had assembled in 0ctober 2005 at the birthplace of Spanish neuroscience pioneer Santiago Ramón y Cajal to discuss the diversity of neocortical interneurons, but soon discovered they weren't even speaking the same language. "In the end, we spent several days locked in a room discussing and essentially listing the features that could be useful to classify cortical interneurons," says Giorgio Ascoli, of the Krasnow Institute for Advanced Study, "and this exercise brought out the general issue of, 'Hey, let's just agree on names that everyone can use."'

The resulting project, the Petilla Interneuron Nomenclature Group, represents an important step toward developing a comprehensive neuron catalog. "Just like an engineer might know that a circuit board has 50 different components, I'd like to go into my brain slices and know if there are 100 different cell types," says Yuste.

Functional diversity of neurons is inextricably linked to structural diversity. "You can actually look at how the shape of the dendrite and axon affect potential synaptic connectivity," says Ascoli, "and if you can characterize potential synaptic connectivity, you can get a good correlate of many computational properties of the network." Accordingly, Ascoli is dedicated to building a 'parts list'. His highly successful NeuroMorpho (http://neuromorpho.org/) project offers scientists a free resource for sharing experimentally derived three-dimensional neuronal reconstructions, with over 5,500 cell structures deposited and more than 400,000 data downloads to date. He is also heading a second project slated for launch in 2010, the Neuron Registry, which will be a centralized database of neuron type-specific characteristics.

These efforts require powerful software tools; popular options like MBF Bioscience's Neurolucida or Molecular Devices' MetaMorph are highly effective for manual or computer-assisted reconstruction, but greater throughput is still needed. "Our hope is that we can come to a point where automated reconstructions will allow us to bring the functional modeling of neurons right into the experiment," says Saggau, whose team recently developed an automated dendrite reconstruction system termed Online Reconstruction and Imaging of Neurons (ORION). To stimulate effort in this area, Ascoli's team has partnered with the Howard Hughes Medical Institute and the Allen Brain Institute to launch the Digital Reconstruction of Axonal and Dendritic Morphology (DIADEM) Challenge (http://diademchallenge.org/), a competition for academic and commercial software experts. "There are five datasets from real-world scenarios that are representative of the kind of work being done in the field," says Ascoli. "If your software can tackle all of them or most of them, you're in a good position."
One's microscope needs depend heavily on the experiments being performed, and both Olympus and Zeiss highlight their systems' flexibility. For those on the frontier of the field, however, pushing the limits of the technology still means gathering or designing lasers, detectors and other paraphernalia and building from the ground up. Yuste offers instructions on his laboratory website (http://www.columbia. edu/cu/biology/faculty/yuste/methods. html). "It's bad, because our labs very often look like engineering workshops," he says, "but it's good because the people who do the experiments know intimately well how every single piece of the machine works, and they can actually improve it to the advantage of the experiment."

\section{Built for speed}

Some of the most exciting applications of two-photon microscopy involve observing neuronal circuits, which requires the capacity both to visualize ultrafine details of cellular morphology (see Box 1) and to monitor individual action potentials and track signal transmission across chains of cells. Although the former goal is essentially within reach, the latter can require serious acceleration of the scanning process.

The laser is typically raster scanned across the sample by galvanometers in a thorough-but potentially wastefulmanner. "The idea is that you want to get away from standard raster-scanning, lineby-line acquisition of images, and rather try to minimize background scan time and maximize scan time on the cells that you're interested in," says Fritjof Helmchen, of the University of Zurich. To achieve this, Helmchen's team devised a smarter, trajectory-based scanning approach, in which the beam is moved along a user-defined threedimensional path within the sample. "With this approach, we could measure from hundreds of cells within a volume with temporal resolution of 10 hertz," he says.

Mechanical scanning systems are fundamentally constrained by inertia, and acousto-optical deflectors (AODs) manufactured by companies like Isomet, IntraAction and AA Opto Electronic — can offer a smoother and faster alternative, in which laser positioning is modulated by a virtual diffraction grating established via propagation of high-frequency sound waves through a transparent material. The angle of light diffraction is governed by the sound-wave frequency and this, according to Baylor College of Medicine neuroscientist Peter Saggau, enables rapid and inertiafree beam movement. Saggau developed a system called random-access multiphoton (RAMP) microscopy that, like Helmchen's technique, allows users to pick multiple destinations in a sample for laser imaging

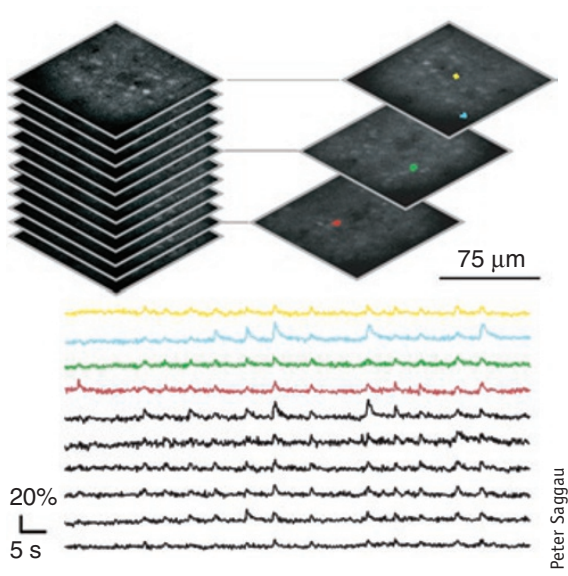

Saggau's three-dimensional RAMP technique enables the tracking of rapid, spontaneous activity from multiple neurons within a brain volume. 

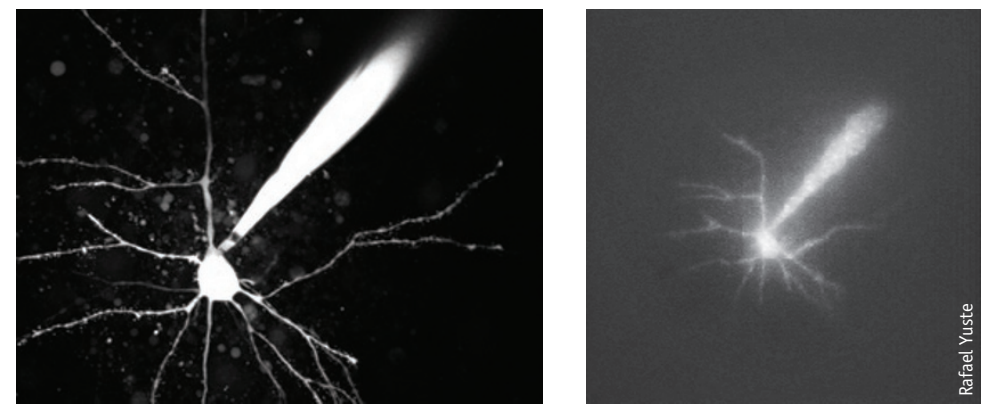

Yuste's team uses neuronal image data collected via two-photon microscopy (left) to generate an SLM illumination pattern (right) that can be used to image or stimulate virtually anywhere on the cell in a scan-free manner.

or stimulation but that uses AODs to make the beam's three-dimensional 'tour' considerably quicker. "We can visit around 50,000 sites per second and can distribute them wherever we want," says Saggau. "If we pick 50 sites on the dendrite of a single neuron or probe multiple neurons, we can scan 1,000 volumes per second."

At the same time, AODs can be unwieldy and require additional effort to minimize beam distortion. Another promising approach currently under development by Yuste and others involves spatial light modulators (SLMs), dynamic computercontrolled optical elements that enable precise patterning of light for scanless multisite imaging or stimulation. "The idea is to move to software the kinds of manipulations that you do with light in hardware," explains Yuste. "We've built a little pocket microscope that literally fits into a shoebox and theoretically has the same capabilities as a giant laser-scanning microscope." His early attempts were nearly too cutting edge- "We used to have significant problems getting technology into our labs because it was military grade," he saysbut commercialization by companies such as HOLOEYE Photonics is making SLMs more accessible.

\section{Penetrating insights}

Most two-photon neuroimaging studies examine living neurons within brain slices, but a growing number of researchers are finding success visualizing the brain directly through the skulls of living animals, via either a thinned cranial patch or an actual transparent 'window'.

According to Simbürger, the Zeiss LSM $7 M P$ was designed for such applications. "Intravital imaging typically requires anesthesia for the mouse, and you have to keep it warm or arrange it in a certain way to get access to the brain," she says. "This requires a lot of space and accessibility 


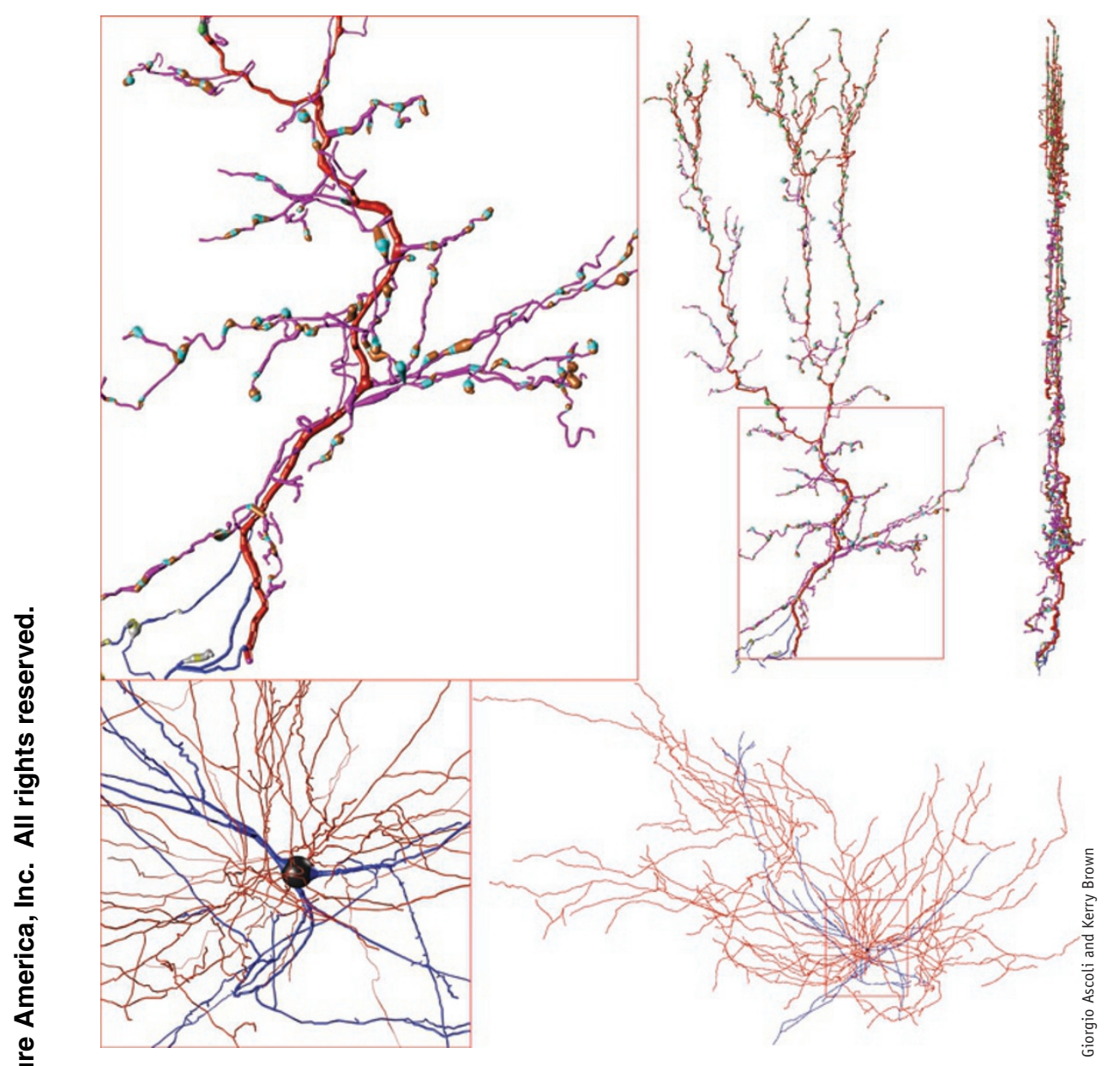

The NeuroMorpho repository contains a rapidly growing collection of three-dimensional neuron reconstructions, including this cerebellar climbing fiber (top) and hippocampal CA3 interneuron (bottom).

to the system." Instead of standard photomultiplier-tube detectors, the LSM $7 \mathrm{MP}$ system can also be upgraded to incorporate gallium arsenide phosphide (GaAsP) detectors—originally developed by Hamamatsu - which offer both enhanced sensitivity and increased signalto-noise ratio, features that can represent a boon to deep-tissue imaging by maximizing detection of scarce photons.

Standard two-photon microscopy can take you deep, but when Mark Schnitzer decided back in 1999 that he wanted to go deeper, he naturally turned to Bell Labs. "They had a wonderful neuroscience group-and since they were the phone company, they had a department of fiber optics," he says. Today, Schnitzer's team at Stanford University has essentially pioneered the field of deep brain imaging via microendoscopy, using fiber-optic systems for epifluorescence and two-photon imag- ing deep inside the brain-even in active animals (see Box 2).

Developing appropriate optics was a serious challenge, and Schnitzer's work was greatly facilitated by the use of gradient refractive index (GRIN) lenses, such as those manufactured by GRINTECH. "We form a refractive index profile inside a glass rod," says Bernhard Messerschmidt, the company's cofounder and chief technical officer. "It's a radial distribution of refractive index, and the nice thing about this is you can make lenses out of these glass rods that don't need curved surfaces any more to enable lens performance." These lenses can be as tiny as 200 microns - perfect for fiber optics-and Schnitzer's team has achieved sufficient resolution to image individual hippocampal dendritic spines in anesthetized animals.

Researchers can also look to Mauna Kea Technologies, whose Cellvizio LAB is the 
only integrated platform currently on the market for endomicroscopic confocal imaging with cellular resolution. "Our probes are only one-third of a millimeter in diameter, to minimize invasiveness," says chief scientific officer François Lacombe. "Any region of the mouse brain can be accessed and observed in vivo, even at a six-millimeter depth." The system offers spatial resolution as low as 1.4 microns and can image a 600-micron-diameter field at 12 frames per second.

\section{BOX 2 ON THEIR BEST BEHAVIOR}

For some studies, monitoring in vivo brain activity under anesthesia is no substitute for working with awake and behaving animals, and two major approaches have emerged for such experiments. The first involves habituating animals to head restraint under a microscope, which limits the behaviors that can be studied but eliminates the need for specialized instrumentation. "You can use these very-large-numericalaperture water-immersion objectives that an animal could never carry," says Schnitzer. "Behavior is much more constrained, but in some cases the constraint might be useful."

The other alternative is to make tiny, fiber optics-based microscopes the animals can wear-a major technical challenge. "I was very focused on bringing imaging to freely moving mice," says Schnitzer. "That's why we really had to go to lengths to miniaturize our instruments to within the 1-3 gram range." Although there are tradeoffs in terms of optics, Schnitzer's team has achieved remarkable success, developing mouse-friendly epifluorescence and two-photon microscopes that take advantage of a combination of microlenses and tiny microelectromechanical systems (MEMS)-based scanning mirrors.

Mauna Kea Technologies is also moving into this arena with the confocal CerboFlex system, slated for launch in 0ctober. "We designed a dedicated implant that permits secure and firm installation of our probes onto the head of an animal," says Lacombe, "and the unprecedented small weight of the deviceless than 1 gram-combined with the extreme flexibility of the probe itself now makes the animal totally free to move."

Although this line of research is still very young, these tools already offer a host of opportunities, and Lacombe hopes his company's system will help expand the ability to perform such studies more routinely. From Schnitzer's perspective, this is a method on the verge of turning an important corner. "It's progressed from technology development to something we use every week in the lab to study mouse behavior," he says. 


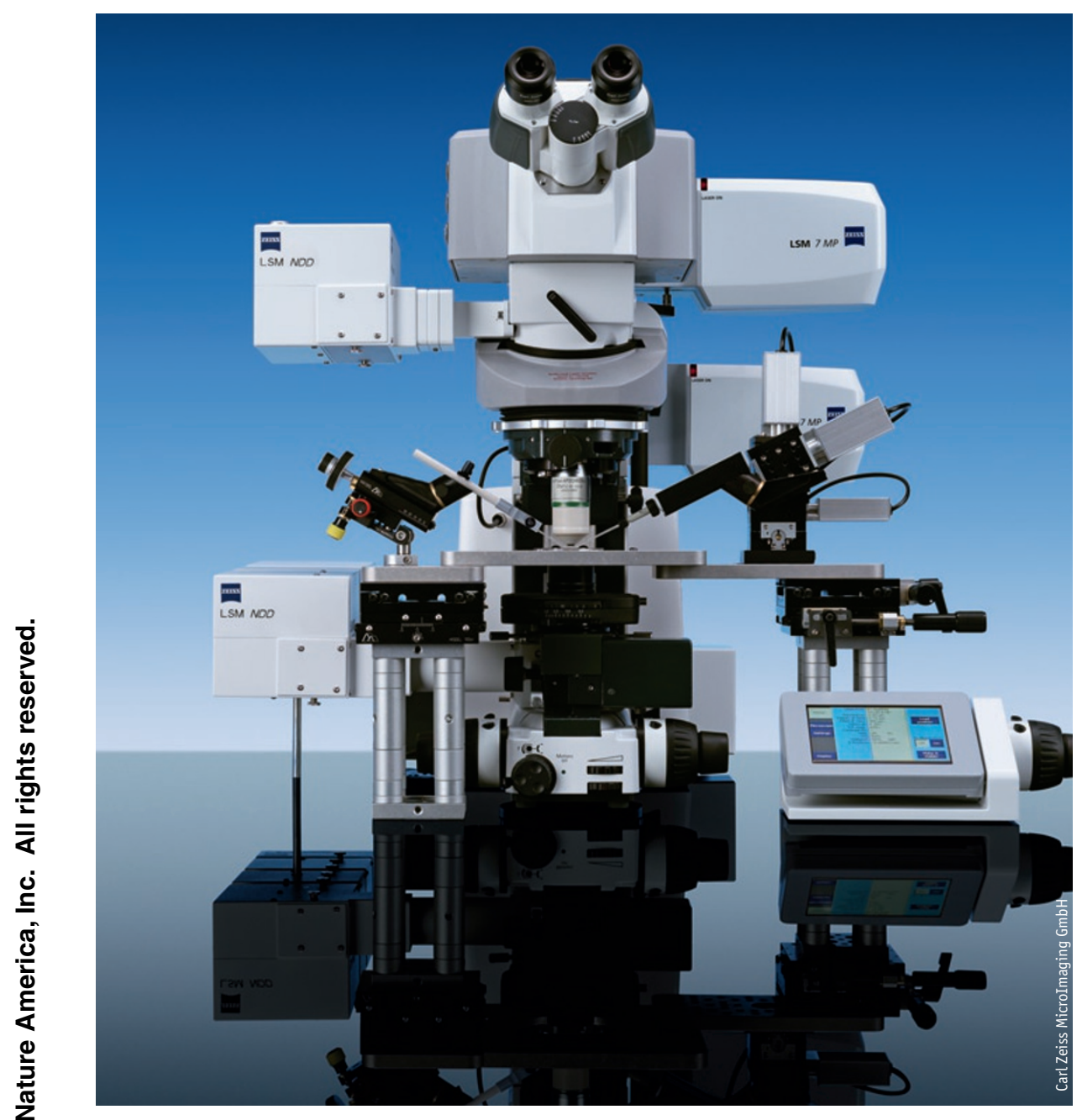

The LSM 7 MP is Zeiss's first purpose-built multiphoton instrument, designed with intravital imaging applications in mind.

\section{Seeing where the action is}

Of course, none of these technologies would be effective without visual indicators of neuronal activity; fortunately, there are numerous well-characterized chemical calcium sensors that can provide rapid and specific readout of action potentials. "Every time a neuron fires, calcium channels open," says Yuste. "It's essentially a universal method to detect action potentials." Invitrogen, a division of Life Technologies Corporation, offers many of the most popular of these dyes, including Oregon Green 488 BAPTA-1 AM and Fluo-4 AM, two membrane-permeable compounds that exhibit increased fluorescence intensity upon binding free $\mathrm{Ca}^{2+}$.

Chemical dyes lack cell-type specificity, but this can be an asset. "Dyes that are readily taken up by responsive neurons via multicell bolus loading are the ones that are enjoying the most success," says Mike
Ignatius, product manager for cell analysis essentials at Life Technologies. "You can fill a population of neurons and then use an electrode to stimulate at a distance and see the postsynaptic response." These dyes can also be directly injected into cells, but this can be

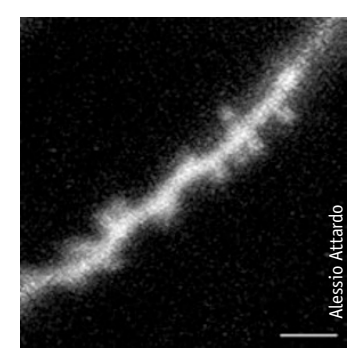

The fiber-optic microscopes developed by Schnitzer's team enable visualization of individual dendritic spines from a CA1 hippocampal pyramidal neuron in a live mouse. Scale bar, $5 \mu \mathrm{m}$. 
painstaking, and genetically encoded calcium indicators (GECIs) may offer a better alternative for labeling specific neuron subpopulations. A few commercial options are available, including Premo Cameleon from Invitrogen and Case12 from Evrogen, and GECIs are now an area of active development. "The use of functional proteins is something that's now really on the verge of working and, in my mind, will completely explode in the next years," says Helmchen.

At the same time, researchers in this field remain painfully aware of the need for direct measurement of action potentials. "Calcium is just the appetizer for the really big thing, which is imaging voltage," says Yuste, "and to be completely honest, there's still nothing out there that compares even closely to the types of data and signal to noise we can obtain with calcium imaging."

\section{All together now}

Each of these leaps represents a starting point for new progress. For instance, many researchers working with the current generation of dyes see their greatest potential in combination with other fluorescent tools; Ignatius specifically highlights examples pairing calcium indicators with signal transduction pathway-specific activity sensors. Meanwhile, others are exploring new imaging modalities as complements or alternatives to two-photon methods, including the use of second-harmonic generation to image membrane potential, or of structured illumination methods.
Microscope manufacturers are likewise seeing opportunities for new systems based on relatively 'frontier' techniques such as super-resolution, as in Leica's TCS STED microscope, or label-free cellular imaging via coherent anti-Stokes Raman scattering (CARS), which Olympus will introduce in October as a module for the FV1000-MPE. Above all, the key will be to find ways to integrate these advances intelligently. "A lot has already happened in terms of progress. .. and now the challenge is to do it all together," says Helmchen, "which sometimes sounds easier than it is!”

Michael Eisenstein is a freelance science journalist based in Brooklyn, New York (incandenza66@gmail.com). 
TECHNOLOGY FEATURE

SUPPLIERS GUIDE: COMPANIES OFFERING MICROSCOPES, MICROSCOPE

COMPONENTS AND SOFTWARE

\section{Company}

AA OptoElectronic

Amerinex Applied Imaging

AnaSpec

Andor Technology

Becker \& Hickl GmbH

Bitplane

Carl Zeiss

Cambridge Technology

Chroma Technology

Coherent

Cooke Corp.

CVI Melles Griot

DVC Co.

eBioscience

Evrogen

Graftek Imaging

GRINTECH GmbH

Hamamatsu Photonics

HOLOEYE Photonics

Improvision

Intelligent Imaging Innovations

IntraAction Corp.

Invitrogen (Life Sciences Corp.)

Isomet

Leica Microsystems

LaVision BioTec GmbH

Mauna Kea Technologies

MBF Bioscience

MDS Analytical Technologies

MediaCybernetics

Newport

Nikon Instruments Inc.

Olympus America

Omega Optical

Optical Imaging

Optronics

PerkinElmer

Photometrics

Physik Instrumente

Prairie Technologies, Inc.

Prior Scientific

QImaging

Quorum Technologies

Schott

Scientific Volume Imaging

Semrock

Sigma-Aldrich

Sutter Instrument

TILL Photonics

Tocris Bioscience

\section{Web address}

http://opto.braggcell.com/

http://www.amerineximaging.com/

http://www.anaspec.com/

http://www.andor.com/

http://www.becker-hickl.com/

http://www.bitplane.com/

http://www.zeiss.de/micro

http://www.camtech.com/

http://www.chroma.com/

http://www.coherent.com/

http://www.cookecorp.com/

http://www.mellesgriot.com/

http://www.dvcco.com/

http://ebioscience.com/

http://www.evrogen.com/

http://www.graftek.com/

http://www.grintech.de/

http://www.hamamatsu.com/

http://www.holoeye.com/

http://www.improvision.com/

http://www.intelligent-imaging.com/

http://www.intraaction.com/

http://www.invitrogen.com/

http://www.isomet.com/

http://www.leica-microsystems.com/

http://www.lavisionbiotec.de/

http://www.maunakeatech.com/

http://www.mbfbioscience.com/

http://www.moleculardevices.com/

http://www.mediacy.com/

http://www.newport.com/

http://www.nikoninstruments.com/

http://www.olympusamerica.com/

http://www.omegafilters.com/

http://www.opt-imaging.com/

http://www.optronics.com/

http://www.perkinelmer.com/

http://www.photomet.com/

http://www.physikinstrumente.com/

http://www.prairie-technologies.com/

http://www.prior.com/

http://www.qimaging.com/

http://www.quorumtechnologies.com/

http://www.us.schott.com/

http://www.svi.nl/

http://www.semrock.com/

http://www.sigmaaldrich.com/

http://www.sutter.com/

http://www.till-photonics.com/

http://www.tocris.com/ 